\title{
PERIODIC SOLUTIONS OF DEGENERATE DIFFERENTIAL EQUATIONS IN VECTOR-VALUED FUNCTION SPACES.
}

\author{
CARLOS LIZAMA AND RODRIGO PONCE
}

\begin{abstract}
Let $A$ and $M$ be closed linear operators defined on a complex Banach space $X$. Using operator-valued Fourier multipliers theorems, we obtain necessary and sufficient conditions to guarantee existence and uniqueness of periodic solutions to the equation $\frac{d}{d t}(M u(t))=A u(t)+f(t)$, in terms of either boundedness or $R$-boundedness of the modified resolvent operator determined by the equation. Our results are obtained in the scales of periodic Besov and periodic Lebesgue vector-valued spaces.
\end{abstract}

\section{INTRODUCTION}

We are concerned with the regularity of solutions to the equation

$$
\frac{d}{d t}(M u(t))=A u(t)+f(t), \quad 0 \leq t \leq 2 \pi,
$$

where $(A, D(A))$ and $(M, D(M))$ are (unbounded) closed linear operators on a Banach space $X$, with $D(A) \subseteq D(M)$. The model (1.1), in case that $A=\Delta$ is the Laplacian and $M=m$ is the multiplication operator by a function $m(x)$, was first considered by Carroll and Showalter [13] and has been recently studied by Marinoschi [26]. This model describes, for example, the infiltration of water in unsaturated porous media, in which saturation might occur. The function $m(x)$ characterizes the porosity of the nonhomogeneous medium, while the fact that $m$ is zero indicates the existence of impermeable intrusions in the soil. A study of solutions for this model, with $m(x)=1$ and periodic initial conditions was made in [27] in case of a nonlinear convection, in connection with some results given in [21]. An interesting analysis of periodic solutions to a nonlinear model consisting in a degenerate diffusion equation of the form (1.1) with homogeneous Dirichlet boundary conditions, where $A$ is a multivalued linear operator, has been given recently in the paper [18].

A detailed study of linear abstract degenerate differential equations, using both the semigroups generated by multivalued (linear) operators and extensions of the operational method from Da Prato and Grisvard has been described in the monograph [17].

2000 Mathematics Subject Classification. 35K65, 34G10, 34K13.

Key words and phrases. Degenerate parabolic equations; Operator-valued Fourier multipliers; $R$ boundedness; $U M D$-spaces; Besov spaces.

The first author is partially supported by FONDECYT Grant \#1100485. 
Regularity of solutions in various vector-valued function spaces for the abstract equation (1.1) with periodic initial conditions

$$
M u(0)=M u(2 \pi),
$$

using the sum method have been studied in [5]. The obtained results gives sufficient conditions for periodicity, but leaves as an open problem to characterize the maximal regularity in terms of hypothesis of the modified resolvent operator $(\lambda M-A)^{-1}$ of the operators $M$ and $A$.

On the other hand, Arendt and $\mathrm{Bu}[3]$, using operator-valued Fourier multiplier theorems, have derived spectral characterizations of maximal regularity in Lebesgue spaces for the equation (1.1) with $M=I$, the identity operator, and periodic initial conditions. Similar characterizations were then obtained for the scale of Besov spaces [4] and subsequently, the scale of Triebel-Lizorkin [7] spaces. See also [23] and references therein. This connection motivates the question whether it is possible to obtain a similar characterization for the problem (1.1)-(1.2). We note that, starting with the work [3], the problem of characterization of maximal regularity for evolution equations with periodic initial conditions have been studied intensively in the last years. See e.g. [8], [9], [10], [23], [24], [28] and references therein. For one side, the main novelty in this paper relies in the presence of two non-commuting operators $A$ and $M$, that are only related by the domain. There are only few papers dealing with this situation (see [25]), and this work can be considered as a progress on the treatment of such kind of problems. On the other side, our approach give immediate application to degenerate evolution equations, arising from applications. Some examples are given in the last section of this paper.

This work is organized as follows: After some preliminaries in the first section, and under some kind of geometrical assumption on the Banach space $X$, we are able to characterize in Section 2 the uniqueness and existence of a strong $L^{p}$-solution for the problem (1.1)-(1.2) solely in terms of a property of boundedness for the sequence of operators $i k M(i k M-A)^{-1}$. We remark that no additional assumption on the operator $A$ is required. In Section 3, we prove a characterization is the context of Besov spaces. We notice that in this case an additional hypothesis on $X$ is not longer required. In the particular case of Hölder spaces $C^{s}((0,2 \pi) ; X), 0<s<1$, we obtain that the following assertions are equivalent in general Banach spaces, provided $D(A) \subset D(M)$ :

(1) $i k M-A$ is invertible for all $k \in \mathbb{Z}$ and $\sup _{k \in \mathbb{Z}}\left\|i k M(i k M-A)^{-1}\right\|<\infty$.

(2) For every $f \in C^{s}((0,2 \pi) ; X)$ there exist a unique function $u \in C^{s}((0,2 \pi) ; D(A))$ such that $M u \in C^{s+1}((0,2 \pi) ; X)$ and (1.1)-(1.2) holds for a.e. $t \in[0,2 \pi]$.

We remark that this result extends and improves [5, Theorem 2.1]. Finally, some concrete examples are examined.

\section{PRELIMinaRies}

Let $X, Y$ be Banach spaces. We denote by $\mathcal{B}(X, Y)$ be the space of all bounded linear operators from $X$ to $Y$. When $X=Y$, we write simply $\mathcal{B}(X)$. For a linear operator $A$ 
on $X$, we denote domain by $D(A)$ and its resolvent set by $\rho(A)$. By $[D(A)]$ we denote the domain of $A$ equipped with the graph norm.

A Banach space $X$ is said to be $U M D$, if the Hilbert transform is bounded on $L^{p}(\mathbb{R}, X)$ for some (and then all) $p \in(1, \infty)$. Here the Hilbert transform $H$ of a function $f \in$ $\mathcal{S}(\mathbb{R}, X)$, the Schwartz space of rapidly decreasing $X$-valued functions, is defined by

$$
H f:=\frac{1}{\pi} P V\left(\frac{1}{t}\right) * f .
$$

These spaces are also called $\mathcal{H} \mathcal{T}$ spaces. It is a well known that the set of Banach spaces of class $\mathcal{H} \mathcal{T}$ coincides with the class of $U M D$ spaces. This has been shown by Bourgain [6] and Burkholder [11].

Definition 2.1. Let $X$ and $Y$ be Banach spaces. A family of operators $\mathcal{T} \subset \mathcal{B}(X, Y)$ is called $R$-bounded, if there is a constant $C>0$ and $p \in[1, \infty)$ such that for each $N \in \mathbb{N}, T_{j} \in \mathcal{T}, x_{j} \in X$ and for all independent, symmetric, $\{-1,1\}$-valued random variables $r_{j}$ on a probability space $(\Omega, \mathcal{M}, \mu)$ the inequality

$$
\left\|\sum_{j=1}^{N} r_{j} T_{j} x_{j}\right\|_{L^{p}(\Omega, Y)} \leq C\left\|\sum_{j=1}^{N} r_{j} x_{j}\right\|_{L^{p}(\Omega, X)}
$$

is valid. The smallest such $C$ is called $R$-bound of $\mathcal{T}$, we denote it by $R_{p}(\mathcal{T})$.

We remark that large classes of classical operators are $R$-bounded (cf. [19] and references therein). Hence, this assumption is not too restrictive for the applications that we consider in this article.

Remark 2.2.

Several properties of $R$-bounded families can be founded in the recent monograph of Denk-Hieber-Prüss [14]. For the reader's convenience, we summarize here from [14, Section 3] some results.

(a) If $\mathcal{T} \subset \mathcal{B}(X, Y)$ is $R$-bounded then it is uniformly bounded, with

$$
\sup \{\|T\|: T \in \mathcal{T}\} \leq R_{p}(\mathcal{T}) .
$$

(b) The definition of $R$-boundedness is independent of $p \in[1, \infty)$.

(c) When $X$ and $Y$ are Hilbert spaces, $\mathcal{T} \subset \mathcal{B}(X, Y)$ is $R$-bounded if and only if $\mathcal{T}$ is uniformly bounded.

(d) Let $X, Y$ be Banach spaces and $\mathcal{T}, \mathcal{S} \subset \mathcal{B}(X, Y)$ be $R$-bounded. Then

$$
\mathcal{T}+\mathcal{S}=\{T+S: T \in \mathcal{T}, S \in \mathcal{S}\}
$$

is $R$-bounded as well, and $R_{p}(\mathcal{T}+\mathcal{S}) \leq R_{p}(\mathcal{T})+R_{p}(\mathcal{S})$.

(e) Let $X, Y, Z$ be Banach spaces, and $\mathcal{T} \subset \mathcal{B}(X, Y)$ and $\mathcal{S} \subset \mathcal{B}(Y, Z)$ be $R$-bounded. Then

$$
\mathcal{S T}=\{S T: T \in \mathcal{T}, S \in \mathcal{S}\}
$$

is $R$-bounded, and $R_{p}(\mathcal{S T}) \leq R_{p}(\mathcal{S}) R_{p}(\mathcal{T})$. 
(g) Let $X, Y$ be Banach spaces and $\mathcal{T} \subset \mathcal{B}(X, Y)$ is $R$-bounded. If $\left\{\alpha_{k}\right\}_{k \in \mathbb{Z}}$ is a bounded sequence, then $\left\{\alpha_{k} T: T \in \mathcal{T}\right\}$ is $R$-bounded.

Given $1 \leq p<\infty$, we denote by $L_{2 \pi}^{p}(\mathbb{R}, X)$ the space of all $2 \pi$-periodic Bochner measurable $X$-valued functions $f$, such that the restriction of $f$ to $[0,2 \pi]$ is $p$-integrable.

For a function $f \in L_{2 \pi}^{1}(\mathbb{R}, X)$ we denote by $\hat{f}(k), k \in \mathbb{Z}$ the $\mathrm{k}$-th Fourier coefficient of $f:$

$$
\hat{f}(k)=\frac{1}{2 \pi} \int_{0}^{2 \pi} e^{-i k t} f(t) d t
$$

for all $k \in \mathbb{Z}$.

We need the following Lemma.

Lemma 2.3. [3] Let $f, g \in L_{2 \pi}^{p}(\mathbb{R} ; X)$, where $1 \leq p<\infty$ and $A$ is a closed operator on a Banach space $X$. Then, the following are equivalent.

(i) $f(t) \in D(A)$ and $A f(t)=g(t)$, a.e.

(ii) $\hat{f}(k) \in D(A)$ and $A \hat{f}(k)=\hat{g}(k)$, for all $k \in \mathbb{Z}$.

The proof of the following Lemma is analogue to [3, Lemma 2.1] and therefore omitted.

Lemma 2.4. Let $M$ be a closed operator, $u \in L_{2 \pi}^{p}(\mathbb{R} ;[D(M)])$ and $u^{\prime} \in L_{2 \pi}^{p}(\mathbb{R} ; X)$ for $1 \leq p<\infty$. The following assertions are equivalent,

(i) $\int_{0}^{2 \pi}(M u)^{\prime}(t) d t=0$ and there exist $x \in X$ such that $M u(t)=x+\int_{0}^{t}(M u)^{\prime}(s) d s$ a.e. on $[0,2 \pi]$;

(ii) $\widehat{(M u)^{\prime}}(k)=i k M \hat{u}(k)$ for all $k \in \mathbb{Z}$.

We also recall the following definition from [3].

Definition 2.5. For $1 \leq p<\infty$, we say that a sequence $\left\{M_{k}\right\}_{k \in \mathbb{Z}} \subset \mathcal{B}(X, Y)$ is an $L^{p}$-multiplier if, for each $f \in L_{2 \pi}^{p}(\mathbb{R}, X)$, there exists $u \in L_{2 \pi}^{p}(\mathbb{R}, Y)$ such that

$$
\hat{u}(k)=M_{k} \hat{f}(k) \text { for all } k \in \mathbb{Z} \text {. }
$$

We finally recall the following results.

Proposition 2.6. [3] Let $X$ be a Banach space and $\left\{M_{k}\right\}_{k \in \mathbb{Z}}$ be an $L^{p}$-multiplier, where $1 \leq p<\infty$. Then, the set $\left\{M_{k}: k \in \mathbb{Z}\right\}$ is $R$-bounded.

Theorem 2.7. [3] Let $X, Y$ be $U M D$ spaces and let $\left\{M_{k}\right\}_{k \in \mathbb{Z}} \subseteq \mathcal{B}(X, Y)$. If the sets $\left\{M_{k}\right\}_{k \in \mathbb{Z}}$ and $\left\{k\left(M_{k+1}-M_{k}\right)\right\}_{k \in \mathbb{Z}}$ are $R$-bounded, then $\left\{M_{k}\right\}_{k \in \mathbb{Z}}$ is an $L^{p}$-multiplier for $1<p<\infty$. 


\section{A CharaCterization ON VECTOR-VALUEd LEBEsGue SPACES}

We consider in this section the degenerate equation

$$
\left\{\begin{array}{l}
\frac{d}{d t}(M u(t))=A u(t)+f(t), \quad 0 \leq t \leq 2 \pi, \\
M u(0)=M u(2 \pi),
\end{array}\right.
$$

where $A: D(A) \subseteq X \rightarrow X$ and $M: D(M) \subseteq X \rightarrow X$ are closed linear operators, $D(A) \subseteq D(M)$, and $f \in L_{2 \pi}^{p}(\mathbb{R}, X), p \geq 1$. For a given closed operator $M$, and $1 \leq p<\infty$, we define the set

$H_{\text {per }, M}^{1, p}(\mathbb{R} ; D(M))=\left\{u \in L_{2 \pi}^{p}(\mathbb{R} ; D(M)): \exists v \in L_{2 \pi}^{p}(\mathbb{R} ; X), \hat{v}(k)=i k M \hat{u}(k)\right.$ for all $\left.k \in \mathbb{Z}\right\}$. If $M=I$, we denote $H_{\text {per }}^{1, p}(\mathbb{R} ; X)$; see [3]. Next, we introduce the following definition.

Definition 3.1. We say that a function $u \in H_{\text {per }, M}^{1, p}(\mathbb{R} ;[D(M)])$ is a strong $L^{p}$-solution of (3.1) if $u(t) \in D(A)$ and equation (3.1) holds for a.e. $t \in[0,2 \pi]$.

We begin with the following result.

Proposition 3.2. Let $A, M$ be are linear closed operators defined on a UMD space $X$. Suppose that $i k M-A$ is invertible for all $k \in \mathbb{Z}$. Then the following assertions are equivalent

(i) $\left\{i k M(i k M-A)^{-1}\right\}_{k \in \mathbb{Z}}$ is an $L^{p}$-multiplier for $1<p<\infty$;

(ii) $\left\{i k M(i k M-A)^{-1}\right\}_{k \in \mathbb{Z}}$ is R-bounded.

Proof. Define $M_{k}=i k M(i k M-A)^{-1}$. By Proposition 2.6 it follows that (i) implies (ii). Conversely, by Theorem 2.7 is sufficient to prove that the set $\left\{k\left(M_{k+1}-M_{k}\right)\right\}_{k \in \mathbb{Z}}$ is $R$-bounded. In fact, we note the following

$$
\begin{aligned}
k\left[M_{k+1}-M_{k}\right]= & k\left[i(k+1) M[i(k+1) M-A]^{-1}-i k M[i k M-A]^{-1}\right] \\
= & k M\left[i(k+1)[i(k+1) M-A]^{-1}-i k[i k M-A]^{-1}\right] \\
= & k M(i(k+1) M-A)^{-1}[i(k+1)(i k M-A)-i k(i(k+1) M-A)] \times \\
& \times(i k M-A)^{-1} \\
= & k M(i(k+1) M-A)^{-1}[-i A](i k M-A)^{-1} \\
= & -i k M(i(k+1) M-A)^{-1}\left[i k M(i k M-A)^{-1}-I\right],
\end{aligned}
$$

where in the last equality we use the identity $A(i k M-A)^{-1}=i k M(i k M-A)^{-1}-I$. Therefore, since the products and sums of $R$-bounded sequences is bounded, by (d) and (g) in Remark 2.2, the proof is finished.

The following is one of the main results in this paper. It corresponds to an extension of [3, Theorem 2.3] in case $M=I$. 
Theorem 3.3. Let $X$ be a UMD space and $A: D(A) \subseteq X \rightarrow X, M: D(M) \subseteq X \rightarrow X$ linear closed operators. Suppose that $D(A) \subseteq D(M)$. Then, the following assertions are equivalent.

(i) For every $f \in L_{2 \pi}^{p}(\mathbb{R}, X)$, there exist a unique strong $L^{p}$-solution of (3.1);

(ii) $i k M-A$ is invertible for all $k \in \mathbb{Z}$ and $\left\{i k M(i k M-A)^{-1}\right\}_{k \in \mathbb{Z}}$ is an $L^{p}$-multiplier for $1<p<\infty$;

(iii) $i k M-A$ is invertible for all $k \in \mathbb{Z}$ and $\left\{i k M(i k M-A)^{-1}\right\}_{k \in \mathbb{Z}}$ is $R$-bounded.

Proof. (i) $\Rightarrow$ (ii) Follows the same lines of [3, Theorem 2.3]. Let $k \in \mathbb{Z}$ and $y \in X$. Define $f(t)=e^{i k t} y$. By hypothesis, there exists $u \in H_{\text {per, } M}^{1, p}(\mathbb{R},[D(M)])$ such that $u(t) \in D(A)$ and $(M u)^{\prime}(t)=A u(t)+f(t)$. Taking Fourier transform on both sides, we have $\hat{u}(k) \in D(A)$ and,

$$
\begin{aligned}
i k M \hat{u}(k) & =A \hat{u}(k)+\hat{f}(k) \\
& =A \hat{u}(k)+y .
\end{aligned}
$$

Thus $(i k M-A)$ is surjective for all $k \in \mathbb{Z}$. Let $x \in D(A)$. If $(i k M-A) x=0$, then $u(t)=e^{i k t} x$ defines a periodic solution of (3.1). In fact, since $u(t)=e^{i k t} x$ we obtain $(M u)^{\prime}(t)-A u(t)=i k e^{i k t} M x-e^{i k t} A x=e^{i k t}(i k M-A) x=0$. Hence $u \equiv 0$ by the assumption of uniqueness, and thus $x=0$. Therefore, $(i k M-A)$ is bijective. We will see that $\left\{i k M(i k M-A)^{-1}\right\}_{k \in \mathbb{Z}}$ is an $L^{p}$-multiplier.

Let $f \in L_{2 \pi}^{p}(\mathbb{R}, X)$, by hypothesis, there exist $u \in H_{p e r, M}^{1, p}(\mathbb{R},[D(M)])$ such that $u(t) \in$ $D(A)$ and $(M u)^{\prime}(t)=A u(t)+f(t)$. Taking Fourier transform on both sides, and using that $(i k M-A)$ is invertible, we have $\hat{u}(k) \in D(A)$ and $\hat{u}(k)=(i k M-A)^{-1} \hat{f}(k)$. Now, since $u \in H_{\text {per, }, M}^{1, p}(\mathbb{R},[D(M)])$ and by definition of $H_{\text {per, }, M}^{1, p}(\mathbb{R},[D(M)])$, there exist $v \in$ $L_{2 \pi}^{p}(\mathbb{R}, X)$ such that $\hat{v}(k)=i k M \hat{u}(k)$ for all $k \in \mathbb{Z}$. Therefore, we have $\hat{v}(k)=i k M \hat{u}(k)=$ $i k M(i k M-A)^{-1} \hat{f}(k)$.

(ii) $\Rightarrow$ (i) Define $M_{k}=i k M(i k M-A)^{-1}$ and suppose that $\left\{M_{k}\right\}_{k \in \mathbb{Z}}$ is an $L^{p}$-multiplier. Let $f \in L_{2 \pi}^{p}(\mathbb{R}, X)$, then, there exist $u \in L_{2 \pi}^{p}(\mathbb{R}, X)$ such that $\hat{u}(k)=i k M(i k M-$ $A)^{-1} \hat{f}(k)$, for all $k \in \mathbb{Z}$. Now by the identity $I=i k M(i k M-A)^{-1}-A(i k M-A)^{-1}$ it follows that

$$
\begin{aligned}
\hat{u}(k) & =i k M(i k M-A)^{-1} \hat{f}(k) \\
& =\left(I+A(i k M-A)^{-1}\right) \hat{f}(k) .
\end{aligned}
$$

So, we obtain $\widehat{(u-f})(k)=A(i k M-A)^{-1} \hat{f}(k)$. Putting $v:=u-f$, we have $v \in$ $L_{2 \pi}^{p}(\mathbb{R}, X)$, and $\hat{v}(k)=A(i k M-A)^{-1} \hat{f}(k)$. Observe that $A^{-1}$ is an isomorphism of $X$ onto $D(A)$ (seen as a Banach space with the graph norm). Therefore, $A^{-1} \hat{v}(k)=$ $(i k M-A)^{-1} \hat{f}(k)$. Let $w:=A^{-1} v$. Since $A^{-1}$ is a bounded operator, we obtain that $w \in L_{2 \pi}^{p}(\mathbb{R}, X), \hat{w}(k) \in D(A)$ and $\hat{w}(k)=(i k M-A)^{-1} \hat{f}(k)$. So, 


$$
\begin{aligned}
i k M \hat{w}(k)-A \hat{w}(k) & =i k M(i k M-A)^{-1} \hat{f}(k)-A(i k M-A)^{-1} \hat{f}(k) \\
& =(i k M-A)(i k M-A)^{-1} \hat{f}(k) \\
& =\hat{f}(k) .
\end{aligned}
$$

Now, observe that we have

$$
\hat{u}(k)=i k M(i k M-A)^{-1} \hat{f}(k)=i k M \hat{w}(k),
$$

for all $k \in \mathbb{Z}$. Therefore, $w \in H_{\text {per }, M}^{1, p}(\mathbb{R},[D(M)])$. Moreover $M w(0)=M w(2 \pi)$, since $w(0)=w(2 \pi)$ and $w(t) \in D(A)$. Since $A$ and $M$ are closed operators and $\widehat{(M w)^{\prime}}(t)=$ $i k M \hat{w}(k)=A \hat{w}(k)+\hat{f}(k)$, for all $k \in \mathbb{Z}$, one has $(M w)^{\prime}(t)=A w(t)+f(t)$ a.e. by Lemmas 2.3 and 2.4. So $w$ is a strong $L^{p}$-solution of (3.1).

Now, to see the uniqueness, let $u \in H_{p e r, M}^{1, p}(\mathbb{R},[D(M)])$ such that $(M u)^{\prime}(t)=A u(t)$. Then $\hat{u}(k) \in D(A)$, and $(i k M-A) \hat{u}(k)=0$, for all $k \in \mathbb{Z}$. Since $(i k M-A)$ is invertible for all $k \in \mathbb{Z}$, we obtain $\hat{u}(k)=0$ for all $k \in \mathbb{Z}$, and thus $u \equiv 0$.

(ii) $\Leftrightarrow$ (iii) Proposition 3.2.

Corollary 3.4. Let $H$ be a Hilbert space, $A: D(A) \subset H \rightarrow H$, and $M: D(M) \subset H \rightarrow H$ closed linear operators satisfying $D(A) \subseteq D(M)$. Then, for $1<p<\infty$, the following assertions are equivalent:

(i) For every $f \in L_{2 \pi}^{p}(\mathbb{R}, H)$, there exists a unique strong $L^{p}$-solution of (3.1);

(ii) $i k M-A$ is invertible for all $k \in \mathbb{Z}$, and $\sup _{k}\left\|i k M(i k M-A)^{-1}\right\|<\infty$.

Proof. Follows from Theorem 3.3, and the fact that in Hilbert spaces the concepts of $R$-boundedness and boundedness are equivalent [14].

The solution $u(\cdot)$ given in Theorem 3.3 actually satisfies the following maximal regularity property.

Corollary 3.5. In the context of Theorem 3.3, if condition (iii) is fulfilled, we have $(M u)^{\prime}, A u \in L_{2 \pi}^{p}(\mathbb{R}, X)$. Moreover, there exists a constant $C>0$ independent of $f \in$ $L_{2 \pi}^{p}(\mathbb{R} ; X)$ such that

$$
\left\|(M u)^{\prime}\right\|_{L^{p}}+\|A u\|_{L^{p}} \leq C\|f\|_{L^{p}} .
$$

Remark 3.6. From the inequality (3.3) we deduce that the operator L defined by:

$$
(L u)(t)=(M u)^{\prime}(t)-A u(t) \text { with domain } D(L)=H_{p e r, M}^{1, p}(\mathbb{R} ; D(M)) \cap L_{2 \pi}^{p}(\mathbb{R} ; D(A)) \text {, }
$$

is an isomorphism onto. Indeed, since $A$ and $M$ are closed, the space $H_{p e r, M}^{1, p}(\mathbb{R} ; D(M)) \cap$ $L_{2 \pi}^{p}(\mathbb{R}, D(A))$ becomes a Banach space under the norm

$$
\|u\| \mid=\|u\|_{p}+\left\|(M u)^{\prime}\right\|_{p}+\|A u\|_{p} .
$$


We remark that such isomorphisms are crucial for the handling of nonlinear evolution equations (see [2]).

\section{Maximal Regularity on the scale of vector-Valued Besov Spaces}

In this section we consider solutions in vector-valued periodic Besov spaces $B_{p, q}^{s}((0,2 \pi) ; X)$, $1 \leq p \leq \infty, s>0$. For the definition and main properties of these spaces we refer to [4] or [22]. For the scalar case, see [12], [29]. Contrary to the $L^{p}$ case, the multiplier theorems established so far are valid for arbitrary Banach spaces; see [1], [4] and [20]. Special cases here allow one to treat Hölder-Zygmund spaces. Specifically, we have $B_{\infty, \infty}^{s}=\mathcal{C}^{s}$ for $s>0$. Moreover, if $0<s<1$ then $B_{\infty, \infty}^{s}$ is just the usual Hölder space $C^{s}$. We summarize some useful properties of $B_{p, q}^{s}((0,2 \pi) ; X)$. See [4, Section 2] for a proof.

(i) $B_{p, q}^{s}((0,2 \pi) ; X)$ is a Banach space;

(ii) If $s>0$, then $B_{p, q}^{s}((0,2 \pi) ; X) \hookrightarrow L^{p}((0,2 \pi) ; X)$, and the natural injection from $B_{p, q}^{s}((0,2 \pi) ; X)$ into $L^{p}((0,2 \pi) ; X)$ is a continuos linear operator;

(iii) Let $s>0$. Then $f \in B_{p, q}^{s+1}((0,2 \pi) ; X)$ if and only if $f$ is differentiable a.e. and $f^{\prime} \in B_{p, q}^{s}((0,2 \pi) ; X)$.

We begin with the definition of operator valued Fourier multipliers in the context of periodic Besov spaces.

Definition 4.7. Let $1 \leq p \leq \infty$. A sequence $\left\{M_{k}\right\}_{k \in \mathbb{Z}} \subset \mathcal{B}(X, Y)$ is a $B_{p, q}^{s}$-multiplier if for each $f \in B_{p, q}^{s}((0,2 \pi) ; X)$ there exists a function $g \in B_{p, q}^{s}((0,2 \pi) ; Y)$ such that

$$
M_{k} \hat{f}(k)=\hat{g}(k), \quad k \in \mathbb{Z} .
$$

The following concept was studied in [23].

Definition 4.8. We say that $\left\{M_{k}\right\}_{k \in \mathbb{Z}} \subset \mathcal{B}(X, Y)$ is $M$-bounded if

$$
\begin{array}{r}
\sup _{k}\left\|M_{k}\right\|<\infty, \sup _{k}\left\|k\left(M_{k+1}-M_{k}\right)\right\|<\infty, \\
\sup _{k}\left\|k^{2}\left(M_{k+1}-2 M_{k}+M_{k-1}\right)\right\|<\infty .
\end{array}
$$

We recall the following operator-valued Fourier multiplier theorem on Besov spaces.

Theorem 4.9. [4] Let $X, Y$ be Banach spaces and let $\left\{M_{k}\right\}_{k \in \mathbb{Z}} \subseteq \mathcal{B}(X, Y)$ be a $M$ bounded sequence. Then for $1 \leq p, q \leq \infty, s \in \mathbb{R},\left\{M_{k}\right\}_{k \in \mathbb{Z}}$ is an $B_{p, q}^{s}$-multiplier.

We next prove the following result, which is the analogue to Proposition 3.2 .

Proposition 4.10. Let $A: D(A) \subseteq X \rightarrow X, M: D(M) \subseteq X \rightarrow X$ be linear closed operators. Suppose that $D(A) \subseteq D(M)$ and $i k M-A$ is invertible for all $k \in \mathbb{Z}$. Then the following assertions are equivalent. 
(i) $\left\{i k M(i k M-A)^{-1}\right\}_{k \in \mathbb{Z}}$ is an $B_{p, q}^{s}$-multiplier for $1 \leq p \leq \infty, s>0$;

(ii) $\sup _{k \in \mathbb{Z}}\left\|i k M(i k M-A)^{-1}\right\|<\infty$.

Proof. (i) $\Rightarrow$ (ii). Follows the same lines as the proof in [?, Proposition 3.4].

(ii) $\Rightarrow$ (i) Define $M_{k}=i k M(i k M-A)^{-1}$. From the identity (3.2) we obtain:

$$
\sup _{k}\left\|k\left(M_{k+1}-M_{k}\right)\right\|<\infty,
$$

proving (4.4). To verify (4.5), we notice:

$$
\begin{aligned}
& k^{2}\left[M_{k+1}-2 M_{k}+M_{k-1}\right]= \\
&=k^{2}\left[i(k+1) M[i(k+1) M-A]^{-1}-2 i k M[i k M-A]^{-1}+i(k-1) M[i(k-1) M-A]^{-1}\right] \\
&=k^{2} M[i(k+1) M-A]^{-1}[i(k+1)[i k M-A]-2 i k[i(k+1) M-A] \\
&\left.+\quad i(k-1)[i(k+1) M-A][i(k-1) M-A]^{-1}[i k M-A]\right][i k M-A]^{-1} \\
&=k^{2} M[i(k+1) M-A]^{-1}[i(k+1)[i k M-A]-2 i k[i k M-A]-2 i k i M \\
&+ i(k-1)[i(k-1) M-A][i(k-1) M-A]^{-1}[i k M-A] \\
&+\left.2 i \cdot i(k-1) M[i(k-1) M-A]^{-1}[i k M-A]\right][i k M-A]^{-1} \\
&= k^{2} M[i(k+1) M-A]^{-1}\left[\left(i(k+1)-2 i k+i(k-1)+2 i M_{k-1}\right)[i k M-A]-2 i k i M\right] \times \\
& \times[i k M-A]^{-1} \\
&= k^{2} M[i(k+1) M-A]^{-1}\left[2 i M_{k-1}[i k M-A]-2 i k i M\right][i k M-A]^{-1} \\
&= k M[i(k+1) M-A]^{-1}\left[2 i k M_{k-1}[i k M-A]-2 i k i k M\right][i k M-A]^{-1} \\
&= k M[i(k+1) M-A]^{-1}\left[2 i k M_{k-1} \cdot I-2 i k i k M[i k M-A]^{-1}\right] \\
&= k M[i(k+1) M-A]^{-1}\left[2 i k M_{k-1}-2 i k M_{k}\right] \\
&= k M[i(k+1) M-A]^{-1}\left[-2 i k\left(M_{k}-M_{k-1}\right)\right] \\
&= k M[i(k+1) M-A]^{-1}\left[-2 i(k-1)\left(M_{k}-M_{k-1}\right)-2 i\left(M_{k}-M_{k-1}\right)\right]
\end{aligned}
$$

Since, we know that $\left\{k\left(M_{k+1}-M_{k}\right)\right\}_{k \in \mathbb{Z}}$ is bounded, and $\left\{M_{k}\right\}_{k \in \mathbb{Z}}$ is bounded by hypothesis, we conclude from the above identity that,

$$
\sup _{k}\left\|k^{2}\left(M_{k+1}-2 M_{k}+M_{k-1}\right)\right\|<\infty .
$$

So, $\left\{M_{k}\right\}_{k \in \mathbb{Z}}$, is $M$-bounded and therefore, by Theorem $4.9,\left\{M_{k}\right\}_{k \in \mathbb{Z}}$ is an $B_{p, q^{-}}^{s}$ multiplier.

Definition 4.11. Let $1 \leq p, q \leq \infty$ and $s>0$. We say that a function $u \in B_{p, q}^{s}((0,2 \pi) ;[D(A)])$ is a strong $B_{p, q}^{s}$-solution of (3.1) if $M u \in B_{p, q}^{s+1}((0,2 \pi) ; X)$ and equation (3.1) holds for a.e. $t \in(0,2 \pi)$. 
The next Theorem is the main result of this section. It extends [4, Theorem 5.1] with $M=I$.

Theorem 4.12. Let $1 \leq p, q \leq \infty$ and $s>0$. Let $X$ be a Banach space and let $A: D(A) \subseteq X \rightarrow X, M: D(M) \subseteq X \rightarrow X$ linear closed operators. Suppose that $D(A) \subseteq D(M)$. Then, the following assertions are equivalent,

(i) For every $f \in B_{p, q}^{s}((0,2 \pi) ; X)$ there exist a unique strong $B_{p, q}^{s}$-solution of (3.1);

(ii) $i k M-A$ is invertible for all $k \in \mathbb{Z}$ and $\left\{i k M(i k M-A)^{-1}\right\}_{k \in \mathbb{Z}}$ is an $B_{p, q}^{s}$-multiplier for $1 \leq p, q \leq \infty$;

(iii) $i k M-A$ is invertible for all $k \in \mathbb{Z}$ and $\sup _{k \in \mathbb{Z}}\left\|i k M(i k M-A)^{-1}\right\|<\infty$.

\section{Proof.}

$($ ii $) \Leftrightarrow($ iii $)$. Follows from Proposition 4.10.

$(i) \Rightarrow(i i i)$. Suppose that for every $f \in B_{p, q}^{s}((0,2 \pi) ; X)$ there exist a unique strong $B_{p, q^{-}}^{s}$ solution of (3.1). Fix $x \in X$ and $k \in \mathbb{Z}$. Define $f(t)=e^{i t k} x$. Then $f \in B_{p, q}^{s}((0,2 \pi) ; X)$. By hypothesis there exist $u \in B_{p, q}^{s}((0,2 \pi) ;[D(A)])$ with $M u \in B_{p, q}^{s+1}((0,2 \pi) ; X)$ such that $u(t) \in D(A)$ and $(M u)^{\prime}(t)=A u(t)+f(t)$ a.e. $t \in(0,2 \pi)$. By Lemma 2.4 we have $i k M \hat{u}(k)=A \hat{u}(k)+x$. Following the same reasoning in the proof of Theorem 3.3 we obtain that $(i k M-A)$ is invertible for all $k \in \mathbb{Z}$. Let $M_{k}:=i k M(i k M-A)^{-1}$. We will see that $\left\{M_{k}\right\}_{k \in \mathbb{Z}}$ is bounded. Using the Closed Graph Theorem, we have that there exist a constant $C$ independent of $f$ such that

$$
\|M u\|_{B_{p, q}^{s+1}((0,2 \pi) ; X)}+\|A u\|_{B_{p, q}^{s}((0,2 \pi) ;[D(A)])} \leq C\|f\|_{B_{p, q}^{s}((0,2 \pi) ; X)} .
$$

Note that for $f(t)=e^{i t k} x$, the solution $u$ of (3.1) is given by $u(t)=(i k M-A)^{-1} e^{i k t} x$. Hence,

$$
\sup _{k}\left\|i k M(i k M-A)^{-1} x\right\| \leq C\|x\| .
$$

$(i i i) \Rightarrow(i)$. Suppose that $(i k M-A)$ is invertible for all $k \in \mathbb{Z}$ and $\left\{i k M(i k M-A)^{-1}\right\}_{k \in \mathbb{Z}}$ is bounded. Define $M_{k}:=i k M(i k M-A)^{-1}$ and $N_{k}:=(i k M-A)^{-1}$ for $k \in \mathbb{Z}$. Since $\left\{M_{k}\right\}_{k \in \mathbb{Z}}$ is bounded, we have by Proposition 4.10 that $\left\{M_{k}\right\}_{k \in \mathbb{Z}}$ is a $B_{p, q}^{s}$-multiplier. Now, we will see that $\left\{N_{k}\right\}_{k \in \mathbb{Z}}$ is a $M$-bounded sequence. First, note that $A$ is an invertible operator, and hence the identity $i k M(i k M-A)^{-1}=A(i k M-A)^{-1}+I$ imply $N_{k}=$ $A^{-1}\left(M_{k}-I\right)$. So, $\sup _{k \in \mathbb{Z}}\left\|N_{k}\right\|<\infty$. Now, observe that

$$
\begin{aligned}
k\left[N_{k+1}-N_{k}\right] & =k\left[(i(k+1) M-A)^{-1}-(i k M-A)^{-1}\right] \\
& =A^{-1} k\left[M_{k+1}-M_{k}\right] .
\end{aligned}
$$

Hence, by (4.6) we get $\sup _{k \in \mathbb{Z}}\left\|k\left(N_{k+1}-N_{k}\right)\right\|<\infty$. In the same way, we have 


$$
\begin{aligned}
k^{2}\left[N_{k+1}-2 N_{k}+N_{k-1}\right] & =k^{2}\left[A^{-1} M_{k+1}-A^{-1}-2\left[A^{-1}\left(M_{k}-I\right)\right]+A^{-1} M_{k-1}-A^{-1}\right] \\
& =A^{-1} k^{2}\left[M_{k+1}-2 M_{k}+M_{k-1}\right] .
\end{aligned}
$$

Therefore, using (4.7), we obtain

$$
\sup _{k}\left\|k^{2}\left(N_{k+1}-2 N_{k}+N_{k-1}\right)\right\|<\infty .
$$

So, $\left\{N_{k}\right\}_{k \in \mathbb{Z}}$ is a $M$-bounded sequence and, by Theorem $4.9,\left\{N_{k}\right\}_{k \in \mathbb{Z}}$ is an $B_{p, q}^{s}$-multiplier. We conclude that $\left\{M_{k}\right\}_{k \in \mathbb{Z}}$ and $\left\{N_{k}\right\}_{k \in \mathbb{Z}}$ are $B_{p, q}^{s}$-multipliers.

Let $f \in B_{p, q}^{s}((0,2 \pi) ; X)$. There exists $u, v \in B_{p, q}^{s}((0,2 \pi) ; X)$ such that $\hat{u}(k)=i k M(i k M-$ $A)^{-1} \hat{f}(k)$ and $\hat{v}(k)=(i k M-A)^{-1} \hat{f}(k)$ for all $k \in \mathbb{Z}$. So, we have $i k M \hat{v}(k)=\hat{u}(k)$ for all $k \in \mathbb{Z}$. By Lemma 2.3 we obtain $(M v)^{\prime}=u$ a.e. Since $u \in B_{p, q}^{s}((0,2 \pi) ; X)$ we have $(M v)^{\prime} \in B_{p, q}^{s}((0,2 \pi) ; X)$, and so, $M v \in B_{p, q}^{s+1}((0,2 \pi) ; X)$. Also, since $(i k M-A)$ is invertible for all $k \in \mathbb{Z}$ and $\hat{v}(k)=(i k M-A)^{-1} \hat{f}(k)$, we have $v(t) \in D(A)$ and $i k M \hat{v}(k)-\hat{f}(k)=A \hat{v}(k)$, for all $k \in \mathbb{Z}$. So, one has $(M v)^{\prime}(t)=A v(t)+f(t)$ a.e. $t \in(0,2 \pi)$ by Lemma 2.3. Uniqueness follows the same way as in the proof of Theorem 3.3 .

Remark 4.13. Note that the Besov spaces $B_{\infty, \infty}^{s}((0,2 \pi) ; X)$ corresponds to the familiar Hölder spaces $C^{s}$, if $0<s<1$. Hence, Theorem 4.12 extends and improves Theorem 2.1 in [5] where $X$ was considered a reflexive Banach space only.

Example 4.14 .

Let us consider the periodic boundary value problem

$$
\begin{aligned}
\frac{\partial(m(x) u)}{\partial t}-\Delta u & =f(t, x), \text { in }[0,2 \pi] \times \Omega \\
u & =0, \quad \text { in }[0,2 \pi] \times \partial \Omega \\
m(x) u(0, x) & =m(x) u(2 \pi, x) \text { in } \Omega,
\end{aligned}
$$

where $\Omega$ is a bounded domain in $\mathbb{R}^{n}$ with a smooth boundary $\partial \Omega, m(x) \geq 0$ is a given measurable bounded function on $\Omega$ and $f$ is a function on $[0,2 \pi] \times \Omega$. The initial value problem $m(x) u(0, x)=v_{0}$ relative to (4.8)- (4.9) has been studied in [15], [16] both in the spaces $H^{-1}(\Omega), L^{2}(\Omega)$ and in $L^{p}(\Omega), p>1$. The periodic problem (4.8)-(4.10) has been studied in $[5]$ in the spaces $H^{-1}(\Omega)$ and $L^{2}(\Omega)$.

Let $M$ be the multiplication operator by $m$. If we take $X=H^{-1}(\Omega)$ then by $[5$, p.38] (see also references therein), we have that there exists a constant $c>0$ such that

$$
\left\|M(z M-\Delta)^{-1}\right\| \leq \frac{c}{1+|z|}
$$


whenever $\operatorname{Re} z \geq-c(1+|\operatorname{Im}(z)|)$. In particular, in the imaginary axis we have $\| M(i k M-$ $\Delta)^{-1}|| \leq \frac{c}{1+|k|}$, for all $k \in \mathbb{Z}$. Therefore, Theorem 4.12 applies immediately, obtaining existence and uniqueness of solutions of (4.8)-(4.10) in periodic Besov spaces, complementing the results in [5]. On the other hand, and because $H^{-1}(\Omega)$ is a Hilbert space, Corollary 3.4 also applies, obtaining that for all $f \in L_{2 \pi}^{p}\left(\mathbb{R}, H^{-1}(\Omega)\right)$ the periodic problem (4.8)-(4.10) has precisely one strong solution $u$ with maximal regularity.

Example 4.15.

Consider, for $t \in[0,2 \pi]$ and $x \in[0, \pi]$, the problem

$$
\begin{aligned}
\frac{\partial}{\partial t}\left(\frac{\partial^{2}}{\partial x^{2}}+1\right) u(t, x) & =-a \frac{\partial^{2}}{\partial x^{2}} u(t, x)-k u(t, x)+f(t, x) \\
u(t, 0)=u(t, \pi)=\frac{\partial^{2}}{\partial x^{2}} u(t, 0) & =\frac{\partial^{2}}{\partial x^{2}} u(t, \pi)=0 \\
\left(\frac{\partial^{2}}{\partial x^{2}}+1\right) u(0, x) & =\left(\frac{\partial^{2}}{\partial x^{2}}+1\right) u(2 \pi, x),
\end{aligned}
$$

where $a$ is positive constant and $-2 a<k<4 a$. If we take $X=C_{0}([0, \pi])=\{u \in$ $C([0, \pi]): u(0)=u(\pi)\}$ and $K$ the realization of $\frac{\partial^{2}}{\partial x^{2}}$ with domain

$$
D(K)=\left\{u \in C^{2}([0, \pi]): u(0)=u(\pi)=\frac{\partial^{2}}{\partial x^{2}} u(0)=\frac{\partial^{2}}{\partial x^{2}} u(\pi)=0\right\},
$$

then we take $M=K+I, A=a M+(k-a) I$. By [5, p.39,Ex.1.2] we have, as in the above example:

$$
\left\|M(i k M-\Delta)^{-1}\right\| \leq \frac{c}{1+|k|}
$$

for all $k \in \mathbb{Z}$. Therefore, Theorem 4.12 applies, and hence for all $f \in B_{p, q}^{s}((0,2 \pi)$, $\left.C_{0}([0, \pi])\right), s>0,1 \leq p, q \leq \infty$ the problem (4.11)-(4.13) has a unique strong solution $u$ with regularity $\frac{\partial^{2} u}{\partial x^{2}} \in B_{p, q}^{s}\left((0,2 \pi), C_{0}([0, \pi])\right)$. In particular, because the class of Besov spaces contains the class of Hölder spaces, our result recover and extends Example 1.2 in [5].

Remark 4.16. Following a similar method of proof, and using the operator-valued Fourier multiplier theorem stated in [7, Theorem 3.2], an analogous result like Theorem 4.12 for the scale of Triebel-Lizorkin spaces can be proved.

\section{REFERENCES}

[1] H. Amann, Operator-valued Fourier multipliers, vector-valued Besov spaces and applications, Math. Nachr. 186 (1997), 5-56.

[2] H. Amann, Linear and Quasilinear Parabolic Problems. Volume I: Abstract Linear Theory. Monographs in Mathematics, vol 89., Birkhäuser, Basel-Boston-Berlin, 1995.

[3] W. Arendt, S. Bu, The operator-valued Marcinkiewicz multiplier theorem and maximal regularity, Math. Z. 240 (2002), 311-343. 
[4] W. Arendt, S. Bu, Operator-valued Fourier multiplier on periodic Besov spaces and applications, Proc. Edim. Math. Soc. 47 (2) (2004), 15-33.

[5] V. Barbu, A. Favini, Periodic problems for degenerate differential equations, Rend. Instit. Mat. Univ. Trieste XXVIII (Suppl.) (1997) 29-57.

[6] J. Bourgain, Some remarks on Banach spaces in which martingale differences sequences are unconditional, Arkiv Math. 21 (1983), 163-168.

[7] S. Bu, J. Kim, Operator-valued Fourier multipliers on periodic Triebel spaces, Acta Math. Sinica (Engl. Ser.) 21 (2005), 1049-1056.

[8] S. Bu, Y. Fang, Maximal regularity of second order delay equations in Banach spaces. Sci. China Math. 53 (1) (2010), 51-62.

[9] S. Bu, Y. Fang, Periodic solutions of delay equations in Besov spaces and Triebel-Lizorkin spaces. Taiwanese J. Math. 13(3) (2009), 1063-1076.

[10] S. Bu, Y. Fang, Periodic solutions for second order integro-differential equations with infinite delay in Banach spaces. Studia Math. 184(2) (2008), 103-119.

[11] D.L. Burkholder, A geometrical condition that implies the existence of certain singular integrals on Banach-space-valued functions. In: Proc. Conference on Harmonic Analysis in Honor of Antoni Zygmund, Chicago 1981, pp.270-286, Wadsworth, Belmont, CA, 1983.

[12] P.L. Butzer, H. Berens, Semi-groups of operators and approximation, Die Grundlehren der Mathematische Wissenschaften, 145, Springer Verlag, 1967.

[13] R. W. Carroll, R. E. Showalter, Singular and degenerate Cauchy problems, Mathematics in science and engineering,127, New York, Academic Press, 1976.

[14] R. Denk, M. Hieber, J. Prüss, R-boundedness, Fourier multipliers and problems of elliptic and parabolic type, Mem. Amer. Math. Soc. 166 (788), 2003.

[15] A. Favini, A. Yagi, Multivalued linear operators and degenerate evolution equations, Ann. Mat. Pura Appl., 163 (1993),353-394.

[16] A. Favini, A. Yagi, Space and time regularity for degenerate evolution equations, J. Math. Soc. Japan, 44 (1992),331-350.

[17] A. Favini, A. Yagi, Degenerate differential equations in Banach spaces, Pure and Applied Math.,215, Dekker, New York, Basel, Hong-Kong, 1999.

[18] A. Favini, G. Marinoschi, Periodic behaviour for a degenerate fast diffusion equation, J. Math. Anal. Appl., 351, (2009), 509-521.

[19] M. Girardi, L. Weis, Criteria for R-boundedness of operator families, Lecture Notes in Pure and Appl. Math., 234, Dekker, New York, 2003, 203-221. 
[20] M. Girardi, L. Weis, Operator-valued Fourier multiplier theorems on Besov spaces, Math. Nachr., 251, (2003), 34-51.

[21] A. Haraux, Nonlinear evolution equations global behaviour of solutions, Lecture Notes in Math., 841, Springer-Verlag, 1981.

[22] V. Keyantuo, C. Lizama, Maximal regularity for a class of integro-differential equations with infinite delay in Banach spaces, Studia Math. 168 (1) (2005), 25-50.

[23] V.Keyantuo, C. Lizama and V. Poblete, Periodic solutions of integro-differential equations in vector-valued function spaces, J. Differential Equations,246 (3) (2009), 1007-1037.

[24] C. Lizama, Fourier multipliers and periodic solutions of delay equations in Banach spaces. J. Math. Anal. Appl. 324(2) (2006), 921-933.

[25] C. Lizama, V. Poblete, Maximal regularity for perturbed integral equations on periodic Lebesgue spaces. J. Math. Anal. Appl. 348 (2) (2008), 775-786.

[26] G. Marinoschi, Functional approach to nonlinear models of water flow in soils, Math. Model. Theory Appl., 21, Springer, Dordrecht, 2006.

[27] G. Marinoschi, Periodic solutions to fast diffusion equations with non- Lipschitz convective terms, Nonlinear Anal. Real World Appl., 10 (2) (2009), 1048-1067.

[28] V. Poblete,Solutions of second-order integro-differential equations on periodic Besov spaces. Proc. Edinb. Math. Soc. 50(2) (2007), 477-492.

[29] H.J.-Schmeisser, H. Triebel, Topics in Fourier Analysis and Function Spaces, Chichester, Wiley, 1987.

Universidad de Santiago de Chile, Departamento de Matemática, Facultad de Ciencias, Casilla 307-Correo 2, Santiago-Chile.

E-mail address: carlos.lizama@usach.cl

E-mail address: rodrigo.ponce@usach.cl 